\title{
Fluid mechanics of additive manufacturing of metal objects by accretion of droplets - a survey
}

\author{
Václav Tesařr ${ }^{1, a}$ \\ ${ }^{1}$ Institute of Thermomechanics v.v.i., Academy of Sciences of the Czech Republic \\ Dolejškova 5, 18200 Praha-Kobylisy, Czech Republic
}

\begin{abstract}
Paper presents a survey of principles of additive manufacturing of metal objects by accretion of molten metal droplets, focusing on fluid-mechanical problems that deserve being investigated. The main problem is slowness of manufacturing due to necessarily small size of added droplets. Increase of droplet repetition rate calls for basic research of the phenomena that take place inside and around the droplets: ballistics of their flight, internal flowfield with heat and mass transfer, oscillation of surfaces, and the ways to elimination of satellite droplets.
\end{abstract}

\section{Introduction}

A new approach to manufacturing of objects has been recently gaining momentum. In contrast to traditional subtractive methods of removing not needed material from the processed product, the new method is additive the basic idea is gradual addition of small particles to the machined product. Historically, the underlying idea of production by addition has never been completely absent - gluing together pieces of wood has always been used to make furniture and bricklayers have always made walls by adding together bricks. In the new approach, however, there are three essential novelties:

- accretion of very small, generally sub-millimetre material volumes,

- moving the accretion location by a servomechanism

- computer control of the process

The main advantage of the additive manufacturing is its universality. There is no need for jigs and special tools or similar auxiliary devices. Each individual product may be specially adapted - by a change in the computer program - to become a unique original.

The additive technology, also called " $3 D$ printing", has been already developed during the past two decades but its use so far was mostly limited to mere demonstrations of the principle. Activities concentrated on developing the computer software for the purpose and demonstrating example products made from soft polymer materials. These materials, of course, made the process easy requiring no high temperatures and no high power levels. Indeed, one of the early demonstrations used a cool process: the traversed laser light induced local polymerisation inside a bath of liquid monomer.

${ }^{\mathrm{a}}$ Corresponding author: tesar@it.cas.cz
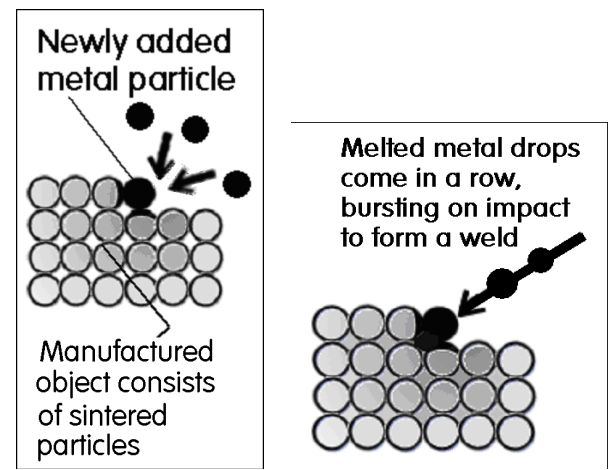

Figure 1 (left) Schematic representation of the additive manufacturing by sintering solid particles. Gaps between the particles are not necessarily a disadvantage - they decrease weight, a factor important e.g. in aeronautical engineering. Figure 2 (right) Schematic representation of the additive manufacturing by melted metal liquid drops that burst upon impact and fill gaps. The surface expecting an impact of the droplet may by also partly melted by local application of heat.

Contemporary mechanical engineering is, of course, heavily dependent on use of metals as the construction materials. If used in the additive manufacturing, metal materials bring quite a number of technological challenges - mainly, of course, associated with the high melting temperatures. There are solutions of these problems, but they have been mostly covered by patents and also need a development of some details. It has to be said that one of the reasons leading to the recent surge of interest in the additive manufacturing is the approaching expiration of patents that were mostly filed at about the turn of the millennium by pioneer inventors.

There are additive production methods that have already gained the status of practical production 
processes, but almost all of them follow the easier approach: the sintering of added solid metal particles (Fig. 1). The particles are supplied in excess to the location where a power beam - mostly laser light - is applied for their partial melting and forming mutual bonds. Advantage of the sintering approach is lower demand on the input power. On the other hand, the sintering bonds between particles are generally less reliable and may result in an incomplete continuity of the inner structure of the product, with the consequence of lower acceptable mechanical loading. Since metals are chosen as the building material mainly to make components that have to carry considerable stresses, the sintering approach has its limitations.

Interest is thus gradually turning towards the more difficult additive manufacturing processes by accretion of sequentially deposited impinging melted metal drops, Fig. 2. The drops are made in a central source and fly to their accretion position by a controlled ballistic curve. They are fixed in position by fusion - the processr encountering problems mainly caused by the high melting temperatures. Some - if not most - among the problems waiting for being solved are of melted metal droplets fluid mechanics.

Particularly challenging tasks are:

- Aero/thermodynamics of droplet flight ballistics,

- Dynamics of surfaces, governed by surface tension,

- Droplet deformation upon hitting its target location,

- Formation of the droplet from a pendant liquid exposed to heating.

\section{Additive method: advantages and problems}

The new additive manufacturing offers a number of attractive advantages. Already mentioned was its universality. Another advantage is much less limits on the complexity of what may be manufactured in a single operation. A particular complexity example in Fig. 3 shows a cross section of a beam with thin curved walls surrounding a half-closed cavity (which may be, without problems, even fully closed. In fact, the method allows producing simultaneously a set of several,

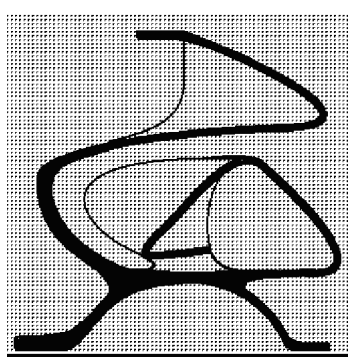

\section{Geometry impossible to make} (requires re-designing)

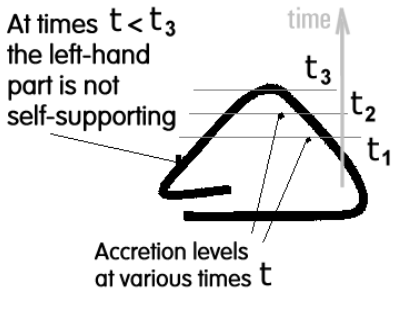

Figure 3 (left) An example of a manufactured component with a very complex cross-section shape. It would be extremely difficult to make it by classical subtractive methods but brings no problem to the additive approach.

Figure 4 (right) The limit to realisability is absence of support during the early stages of additive production.
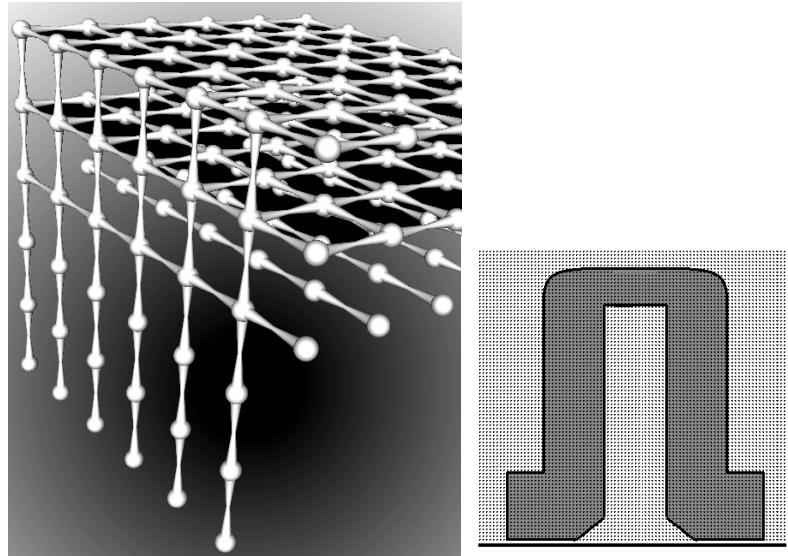

Figure 5 (left) Grids - like this spatial filigree periodic structure - are typical objects that may be quite easily and fast manufactured by the additive method.

Figure 6 (right) An example of a mechanical component with cross section not suitable for the additive method. The simple and wide cross section requires much material the transfer of which makes the additive method unable of competing with the traditional production approaches

mutually disconnected components - such as, e.g., a lock and its key, perhaps with the key positioned partly inside the lock. The number of simultaneously made mutually disconnected components placed inside one another is unlimited: already demonstrated was producing in a single operation a complete gearbox including all its internal gears inside. Obviously, this calls for a completely new approach to mechanical component design, not needing access windows to the internal space or fixing by screws.

On the other hand, it must not be forgotten that additive manufacturing is generally very costly - and is very likely to remain so in foreseeable future. Lasers and other generators of controlled power transfer are often extremely expensive. The other problem is the manufacturing cannot be fast. It would be therefore uneconomical to apply the method to making simply shaped objects, like the girder beam the cross section of which is shown in Fig. 6. Products suitable for the new approach have typically relatively thin curved walls perhaps warped or twisted (Fig. 3) with little limitation to complexity - and objects with internal cavities. Making thin walls, of course, is preferable as it requires a smaller total number of deposited drops and hence a faster production. Adding tiny droplets to cover a large crosssection area in Fig. 6 would be wastage of time and effort.

The general request for increasing production speed leads to development activities concentrating on highfrequency of molten metal formation. Very much depends on requested surface smoothness of produced objects. The higher is the required surface quality, the smaller must be the accreted droplets and the longer takes the manufacturing process. At the current top speed, additive manufacturing has already demonstrated capability to add 200 cubic inches of metal per hour - in other words, $1610^{3} \mathrm{~mm}^{3} / \mathrm{h}$ (or $4.5 \mathrm{~mm}^{3} / \mathrm{s}$ ). As a general rule, by decreasing the size of the droplets to one half the 

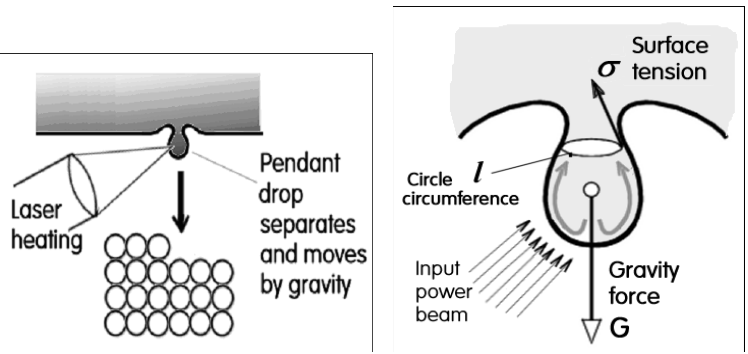

Figure 7 (left) The drop of molten metal is often produced by focused high power beam - in this case a beam of laser light.

Figure 8 (right) Detail of the effects that influence the formed pendant droplet generated by irradiation of a small part at the bottom of supplied metal body.

manufacturing time increases 8-times. Some improvement in the productivity may be obtained by operating two (or more) droplet generating units simultaneously - but apart from the obvious considerable increase in the system price, not all manufactured geometries are suited for the simultaneous operation of two sub-systems without mutual interference of the deposition trajectories or servomechanism components.

\section{Producing the molten metal droplet}

There are two approaches to droplet formation:

- The metal may be melted in a larger quantity in advance and stored in a vessel from which a tiny amount is taken by some ON-OFF valve when needed (Fig. 11). Because of the energy demand for melting and maintaining the molten state - as well as difficulties associated with the valve design - this approach is chosen for metals with lower melting temperatures.

- Otherwise the drop is produced "on demand" - as represented schematically in Fig. 7 - by high power heating pulse applied to the metal material at an end of a wire or at an edge of a strip. Some mechanism has to translate the material to the irradiation location.

Because of the high density of most metals, it is common to generate the drop somewhere above the produced object, so that transporting the molten droplet to its final location may be in principle by gravity. However, small droplet size, high requested repetition rate, and high speed of droplet flight make the gravity action insufficient. It may perhaps dominate the separation of the droplet, Fig. 9, but the subsequent ballistics usually uses other forces. Velocities of droplet flight are nowadays in the range $2-4 \mathrm{~m} / \mathrm{s}$ - with a strong trend towards higher values. In principle the heating associated with impact on target surface might be used for melting the arriving particle, but this is rarely if ever used because the velocities necessary for significant melting effect would call for velocities at least a decimal order of magnitude higher than the above mentioned contemporary values.

Conditions governing the droplet formation "on demand" by input heating power are presented in Fig. 8 . Most important is the surface tension which has to be overcome to separate the droplet and let it move.

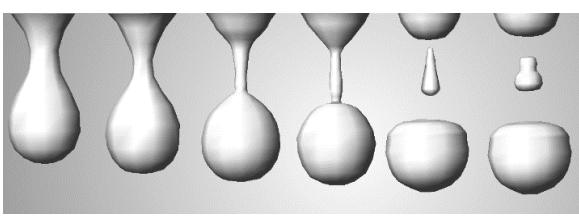

Figure 9. Typical drop formation process (time increasing from left to right). Top of the drop contracts into ligature which, after the drop has pinched off, becomes the unwelcome satellite droplet.

The surface tension, characterised by quantity $\sigma[\mathrm{N} / \mathrm{m}]$, is identical to mechanical energy per unit of surface that must be input to cause the separation. The simplest expression for the force balance of the gravity force $G$ $[\mathrm{N}]$ and surface tension force, neglecting unsteady effects, viscous, and other forces would be

$$
\mathrm{G}=\sigma l
$$

- where $l[13]$ is the circumference of the horizontal cross-section through the "neck" of the formed droplet. The balance is unstable and the separation is facilitated by the surface tension tending to squeeze the liquid into the spherical shape characterized by the least surface energy.

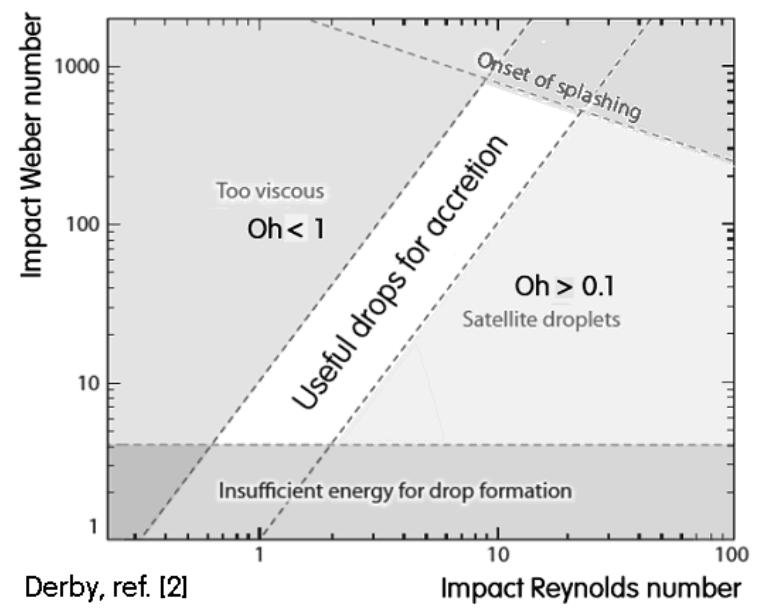

Figure 10. Parameters of metal drops suitable for use in additive manufacturing have to be adjusted to the relatively narrow range of formation conditions.

As shown in Fig. 9, the "neck" contracts into a treadlike ligature, which finally (fourth drop from left in Fig. 9) breaks so that the droplet may begin its ballistic flight. This picture also shows one of the aspects of drop formation that has been a subject of detailed research: the broken ligature under the effect of the minimised surface energy gets transformed into a smaller droplet, called a "satellite". Because of its smaller size, the character of satellite trajectory diffes from that of the main droplet. This flight to a different spot on the impact surface deteriorates the surface quality. Formation of satellite droplets depends on the magnitude of Ohnesorge number

$$
\mathrm{Oh}=\frac{\nu}{\sqrt{\sigma \mathrm{vd}}}
$$




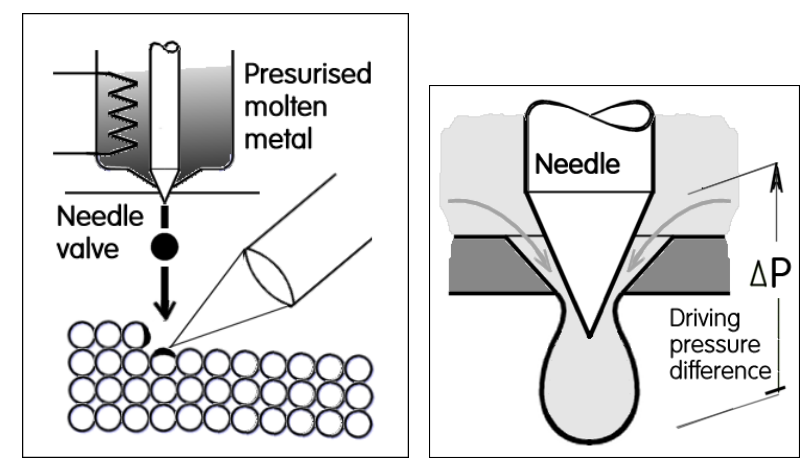

Figure 11 (Left) Generation of a molten metal drop by opening an exit from a bath of metal kept there in the liquid state.

Figure 12 (Right) Detail of producing the drop from the molten metal bath. Hydrodynamics of the flow past the needle tip is discussed in ref. [1]

- where $v\left[\mathrm{~m}^{2} / \mathrm{s}\right]$ is molten metal viscosity (kinematic), $\mathrm{v}\left[\mathrm{m}^{3} / \mathrm{kg}\right]$ is its specific volume, and $\mathrm{d}[13]$ is drop diameter. This dimensionless parameter eq. (2) was introduced by Ohnesorge in 1936 in his $\mathrm{PhD}$ thesis [2]. As a general rule, its is recommended to adjust the drop formation conditions so that the Oh values are within the limits $\mathrm{Oh}>0.1$ and $\mathrm{Oh}<1$ as presented in Fig. 10 (it should be noted that these limit values are based on ref. [3] where they were actually derived for inkjet printing., where the droplet formation conditions may be different). In the diagram Fig. 10 there are also other limits, derived from experimental data on droplet impact behaviour.

A specific study deserves the drop formation in the case of metal melted in advance and stored in the melted phase (Fig. $11-[4,5]$ ). Drop is produced on demand by brief opening of a valve. Figure 12 presents a typical example of the valve performing the flow control by axial motion of the needle. The force balance on the drop is here complicated by the additional pressure forces which ref. [1] shows to be far from simple.

\section{High-energy beams}

\subsection{Flame: a beam of molecules produced by combustion}

The easiest way how to generate the required beam of energetic particles - in this case consisting of molecules produced by a chemical reaction - is to use a burning flame. It may have its merits, such as low cost. It may be particularly suitable - as shown schematically in Fig. 13 - for pre-heating the target are before the droplet impact. Here it is, however, discussed mainly for completeness of the survey, because for the additive manufacturing it has considerable disadvantages.

The highest available temperature, up to $3.5 \mathrm{kK}$ (kilokelvins) is found in the primary flame, using the supplied $\mathrm{O}_{2}$. This generates roughly $2 / 3$ of total heat production. The rest $\sim 1 / 3$ is provided by the outer, secondary flame. One of the disadvantages is flames in general do not reach very far. The length of the very hot region in front of the nozzle is comparable with the nozzle exit diameter. As shown in Fig. 13, the fuel gas is usually brought in by the central nozzle and the oxygen through the outer annular nozzle. The secondary flame uses additional oxygen from the atmosphere. This mixing with atmospheric air decreases the temperature, often to only $\sim 1 \mathrm{kK}$ at the tip of the secondary (outer) flame. Also, the flame widths are generally large compared with the size of typical droplets to be melted. This makes this approach to heat generation rather ineffective for the discussed purposes. A maximum achievable value of thermal power density on the heated surfaces is approximately $\sim 0.1 \mathrm{GW} / \mathrm{m}^{2}$.

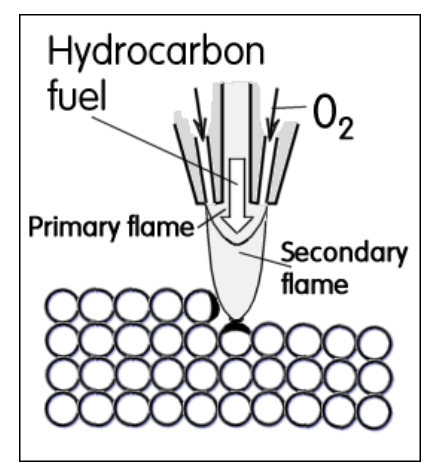

Figure 13. A relatively low-intensity power transfer beam may be generated by exothermic chemical reactions - burning gas in a torch, generally with poor focusing on a small droplet.

\subsection{Plasma torch: a beam of ionised gases}

More powerful than the chemical reaction - and generally better suited for producing the thermal input into drops (and droplets) for their melting are plasma torches. They use energy supplied from an external source in the form of electricity. There are too many alternative variants of plasma torch designs to discuss them all in this survey in detail (most of them were developed, anyway, for a different purpose - metal cutting and welding).

In essence, the impinging heating flow is here plasma (or hot gas with a certain plasma contents) generated by the voltage difference between two electrodes (Fig. 14). One of the electrodes, the cathode, is positioned upstream. Downstream, at or near the exit is a hollow anode. Plasma generated by arc was introduced in 1957 by Dr. R. Gage whose Patent [6] gave for 17 years a virtual monopoly to Union Carbide, Gage's employer. Meanwhile, Prof. Browning [7] and his graduate student Thorpe were developing their own plasma torches concentrating on the task of obtaining high temperature. They managed to achieve $5.6 \mathrm{kK}$, more than the temperature of sun photosphere. The gas that is ionised to become the power carrier for metal heating is mostly an inert one, such as $\mathrm{Ar}$, because even tungsten electrodes would erode rapidly in the presence of oxygen. Much welcome was discovery by Soviet researchers [8,9] of hafnium and zirconium electrodes, capable of working with air. The jet, issuing from the orifice in the anode, is narrow due to electric gradient. Since this means the temperature may be concentrated in a small cross-section area on jet axis, plasma torches may reach high power density, generally as high as $5 \mathrm{GW} / \mathrm{m}^{2}$. 


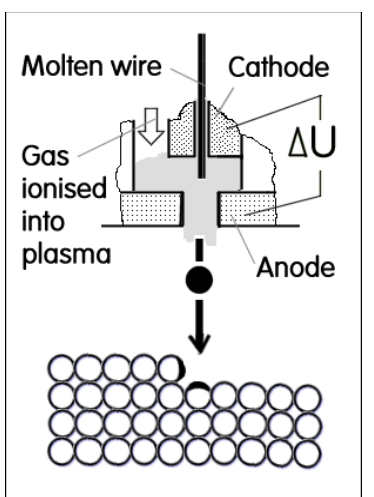

Figure 14. Schematic representation of typical plasma torch. A beam of ions is produced by voltage difference $\Delta U$ exceeding the dielectric breakdown strength of the supplied gas. The hot flow generated by the arc may be easily accelerated either by the gas pressure and/or by the voltage difference.

\subsection{Laser light: photon beams}

Lasers generate beams of photons emitted by a process of optical amplification. Because of the high coherence, the generated laser light beam may remain narrow over long distances - and may be effectively focused by an optical system to a very small illuminated spot. Also here are many existing variants. The best solution for industrial uses is offered by $\mathrm{CO}_{2}$ lasers, invented by Patel [10]. They can continuously emit many hundreds of watts in a single spatial mode in the thermal infrared at $10.6 \mu \mathrm{m}$. It is possible to reach power density values of the order 10 $\mathrm{GW} / \mathrm{m}^{2}$. Important is the fact that efficiency of $\mathrm{CO}_{2}$ lasers is quite high, over $30 \%$.

Very important is the capability of lasers to operate in the mode of extremely short pulses. The table-top sized multi-pass laser described in [11] generates at infra-red in picosecond pulses a power $1.4 \mathrm{~kW}$. Ref. [12] discusses picosecond and femtosecond regime ( $\left.1 \mathrm{fs}=10^{-15} \mathrm{~s}\right)$ pulsed lasers processing components by the additive manufacturing of Ni- and Ti- base alloys. Due to the short pulse duration, power density may easily reach values above $10 \mathrm{TW} / \mathrm{m}^{2}$. Lasers thus can easily melt metal drop-

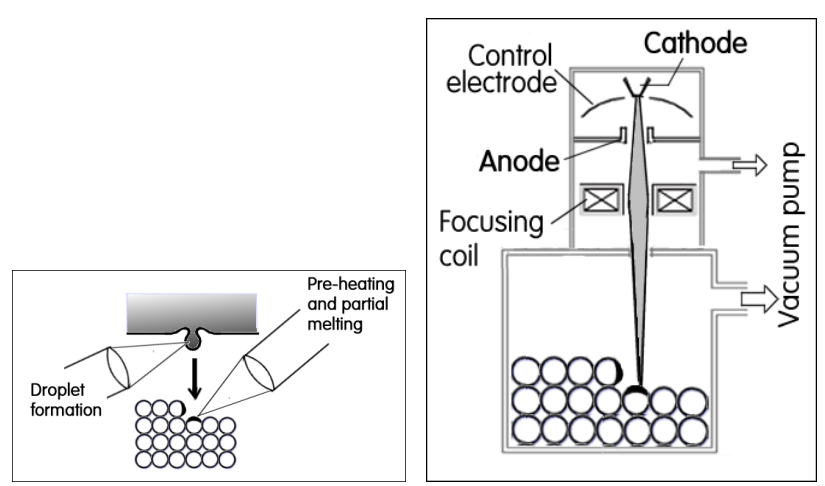

Figure 15 (left) Formation of strong bond with the newly added drop may require re-heating of the target area - perhaps with partial melting, cf. Fig. 2, so that the drop impact may be a case of liquid-liquid interaction.

Figure 16 (right) A particularly high heating power can be carried by a beam of electrons, with the advantageous capability of exact focusing to very small droplet surface area. A disadvantage is the need to operate in vacuum. lets so as to form a dense chain (Figs. 17, 18) - the drop density limited only by the physical dimensions of the drops. Unfortunately, not only the laser itself but also its necessary accompanying facilities tend to be very expensive.

\subsection{Electron beams}

The inventor of this approach to generation of power carrying beams - in this case beams of electrons - was Pirani [13] working for Siemens in Germany. Electrons possess extremely small mass $m=9.1 \cdot 10^{-31} \mathrm{~kg}$ and a negative electric charge $e=1.6 \cdot 10^{-19} \mathrm{C}$. Free electrons in vacuum may be thus very much accelerated by high voltage, usually applied in the range from $\Delta \mathrm{U}=10 \mathrm{kV}$ to $1 \mathrm{MV}$, in narrow beams carrying high kinetic energy transformed into heat upon the impact on solids.

The extraordinary values of handled energy, difficult to compare with common phenomena, has led in this field to introduce an auxiliary unit (not $\mathrm{SI}$ ) $\mathrm{eV}=$ electron volt. It is approximately equal to $1.610^{-19} \mathrm{Ws}$. For comparison, photons of visible light carry $1.5-3.5 \mathrm{eV}$, the electron gun in a cathode ray display tube operate with approximately $20 \mathrm{keV}$, and energy of medical $\mathrm{X}$ rays is typically $0.2 \mathrm{MeV}$. Electrons are emitted from cathode (Fig. 16). They are the conduction electrons in cathode metal (i.e. not bound to atoms). Leaving the cathode requires getting kinetic energy higher than the potential barrier - which e.g. in tungsten is $4.5 \mathrm{eV}$ (often used cathode material is lanthanum hexaboride $\mathrm{LaB}_{6}$, with the low barrier level around $2.5 \mathrm{eV}$ ). Thermionic cathodes use the fact that following the Richardson's rule, the number of electrons possessing the required kinetic energy increases with metal temperature. Another approach is field emission electron gun applying extremely high electric field gradient, typically $10^{\circ} \mathrm{V} / \mathrm{m}$. The beam of electrons may be shaped into a narrow cone by magnetic "lenses" - suitably configured coils.
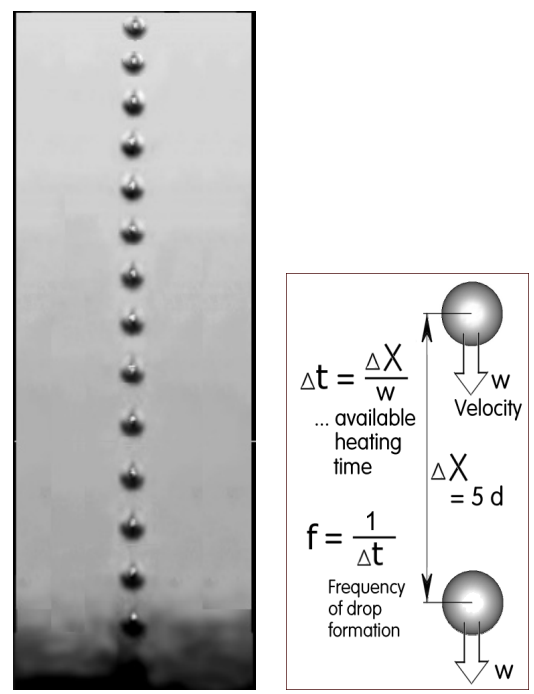

Figure 17 (left) Droplets falling in a chain. Requirements of increasing the manufacturing speed may necessitate working with chains of similar density.

Figure 18 (Right) Basic relations of a droplet chain. The distance of five drop diameters shown in this picture between the chain members may be typical. 
Of course, considerable practical disadvantage of the electron gun is the fact that the electrons can move over the distances of interest only in vacuum - which complicates and slows down the placing of the processed object into its position.

\section{Kinetics of metal drop motion}

Behaviour of molten metal drops in their use for additive manufacturing presents many unanswered questions. Obtaining a deeper understanding is obviously necessary for making the " $3 D$ printing" more efficient and therefore more widely applicable. There is also the question of higher surface quality of the manufactured object without decreasing excessively the drop size.

Because of the complexity and at the same time difficulties associated with experimental investigations of high temperature molten metals, it may be convenient -at least in the initial stages - to simplify the analysis by separating two aspects:

- The fluid mechanics of metal drop motion, and

- Thermomechanics of heat and mass transfer between the drops, their environment (either gas atmosphere or vacuum), and finally the surface on which they are deposited.

\subsection{Ballistics of droplet flight}

Fluid mechanics of drop motion must take into account several important specific facts. One of them is the oscillation that was observed to take place immediately after the drop separation from its source. This may endanger the required high precision of drop motion trajectory - in a similar manner as the oscillation influences the motion of the complementary objects bubbles in liquid $[14,15]$. The importance of the initial part of the droplet trajectory increases with the demands of productivity resulting in the trajectories being rather short. In spite of the rapid viscous damping, because of the generally low Reynolds numbers, the oscillation can significantly influence the initial stages of the separated drop - and these stages are very important because of the general demand to shorten the length of the droplet flight trajectory due to the demands of increased productivity.

Also because of the demands of higher productivity, there is a strong tendency to produce and eject the drops at very high repetition rates. The presence of neighbor drops may influence significantly their trajectories. The drops are likely in near future to move in chains, with rather short distances between the chain members, as is shown in Fig. 15. This means the studies of an individual drop flight, while certainly a useful starting point, are no more than just a preliminary to studies of the motion in chains [4] - especially in those cases where the drops fly in gas atmosphere and thus move in the wake of the predecessor drop. In present-day additive manufacturing systems the frequency of drop generation (Fig. 19) is not particularly high - typically of the order from $2 \mathrm{~Hz}$ to 10 $\mathrm{Hz}$ - but already foreseen are requirements of working with frequencies higher than these by a decimal order of magnitude. This results in very short times $\Delta t$, less than
$0.1 \mathrm{~s}$, that is available for delivering into the forming drop the heat for its melting.

Admittedly, at present the velocities of drop ballistic motion in additional manufacturing facilities are not very high; characteristic values being let us say between $2 \mathrm{~m} / \mathrm{s}$ and $4 \mathrm{~m} / \mathrm{s}$. This motion speed determines the pitch distance $\Delta \mathrm{X}$ between the drops flying in a chain - the value $\Delta \mathrm{X}=5 \mathrm{~d}$ in Figs. 18 and 19 is here chosen as very probably characteristic for the conditions in the very near future. Later condition have to be expected. even higher values The hydrodynamic drag of droplets in a dense chain differ significantly from the usually considered Stokes' law.

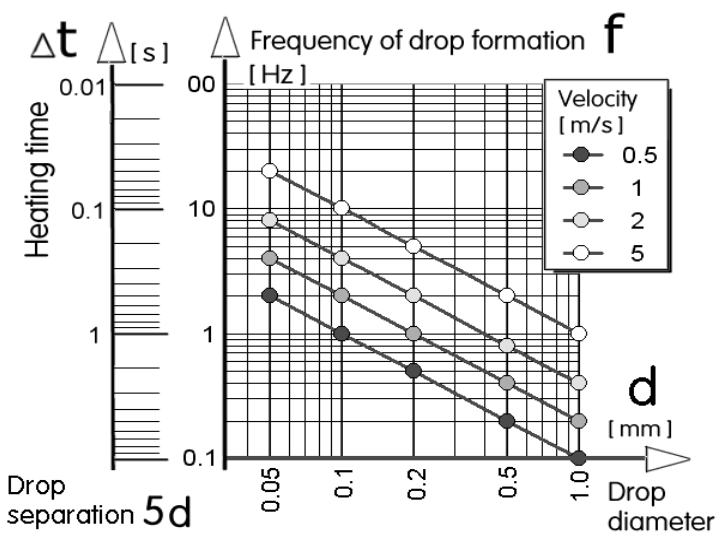

Figure 19. Diagram useful for providing an idea about the drop velocity, frequency of their generation, and the time available for their heating and melting.

\subsection{Dynamics of impact}

Important mechanism influencing proper deposition of the metal from the drop to the target area is the dynamics of droplet impact on its target location. This is a complex problem calling for deeper fluid mechanical studies. There are some not very numerous papers (such as, e.g. [3] and [16]) analyzing the drop impact processes - but their interest was directed towards a different branch of technology: the impact of ink drops on paper, investigated because of the needs of developed inkjet

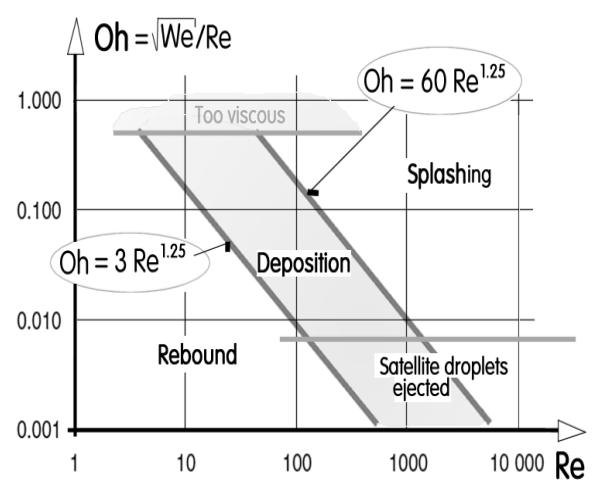

Figure 20. Conditions at the impact should be adjusted so that its dimensionless parameters fit into the deposition region shaded in this diagram. The splashing regime is particularly unwelcome since the impinging droplet there is deformed into a thin layer on the impingement surface rather than a blob suitable for the role of building a structure. 
printers. Some, predominantly qualitative information may be transferable from there, but the need of detailed analysing the process for the purposes of manufacturing of metal objects is obvious.

One of the aspects that may significantly change the character of the impact is the quite often applied heating of the target area, Fig. 16, especially if the heating is at the (quite desirable) level at which the target surface becomes melted. The impact can then get the character of liquid/liquid interaction [17], with the splattered droplet generating the well known "milk crown" configurations, many times reproduced in early high-speed photography.

On the other boundary in Fig. 20, the one opposite to the splashing limit, the proper deposition of the drops may be influenced again by the shape oscillation, Fig 20 - some information about which is known from ref. [14].

It is now well established that the character of the drop impact is determined by the pair of dimensionless characterisation number, the impact Reynolds number:

$$
\operatorname{Re}=\frac{W_{\text {imp }} \mathrm{d}}{\nu}
$$

where $\mathrm{w}_{\text {imp }}[\mathrm{m} / \mathrm{s}]$ is the velocity of the drop prior to the impact, and the Weber number of impact:

$$
W e=\frac{W_{\text {imp }}^{2} \mathrm{~d}}{\sigma \mathrm{V}}
$$

Substantial role in the dynamics of the motions - such as, e.g., of the rebound oscillation (Fig. 2) - has the characteristic impact time:

$$
t_{\text {imp }}=\frac{W_{\text {imp }}}{d}
$$

The splattering or splashing is an unwelcome phenomenon for the additive manufacturing because it reduces the height of the added material and thus reduces the manufacturing speed. It is also likely to aggravate the quality of generated surface, especially at high Reynolds numbers of the impinging motion. As shown in Fig. 20 that combines some aspects of available information about the impact, there is a regime suitable for the drop deposition, the boundaries of which are mainly dependent on the value of Ohnesorge number, eq. (1).

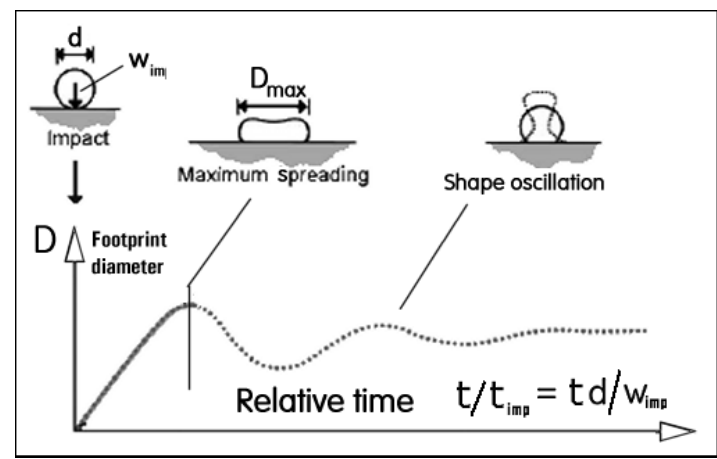

Figure 21 Shape oscillation in the rebound regime is governed by surface energy (and also by viscosity that determines damping of the oscillation). Conditions correspond to those discussed in [14].

\section{Droplet thermomechanics}

The unusual term "thermomechanics" is here used due to the fact that the unsteady thermodynamics of generated drop, cooled during flight and finally solidified involves several aspects of the process, involving also thermokinetics of heat transfer across the droplet surface. Moreover, unless the processes take place in vacuum, there are present also chemical changes because the drops tend to be very soon covered by a layer of oxides.
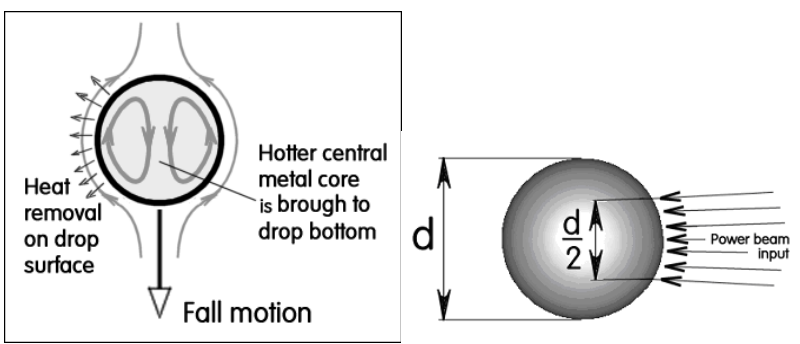

Figure 22 (left) Thermal processes inside a falling drop are influenced by the internal flowfield. Also important is the fact that many (perhaps most) most molten metal drops in air atmosphere are rapidly covered by a layer of oxides.

Figure 23 (right) Simple model of irradiation of a drop used for plotting the diagram Fig. 25

The heat transfer, as suggested in Fig. 22, is also strongly dependent on the difficult to access internal flowfield inside the drop - partly perhaps generated by the skin friction and partly by the inertia of the internal flows in the drop during its formation and separation from the solid mother metal.

Of particular importance are also the extraordinary magnitudes of the radiation heat transfer, involving the extreme devices discussed in Sect. 4. Their high intensities means the governing laws are quite likely to involve nonlinear terms, usually neglected in the relations of standard thermokinetics.

In this paper of survey character, details of these problems cannot be discussed. It is, nevertheless, useful to list them and to provide some basic data for order-ofmagnitude considerations. The essential question that may be asked is the magnitude of thermal energy that has to be delivered into the metal particle so that it is melted and becomes the droplet. The essential information is presented in the diagram Fig. 24. For a range of drop diameters and several representative metals are there plotted thermal input data evaluated from the quantities

\begin{tabular}{|c|c|c|c|}
\hline & $\begin{array}{l}\text { Melting } \\
\text { temperature } \\
{\left[{ }^{\circ} \mathrm{C}\right]}\end{array}$ & $\begin{array}{c}\text { Specific } \\
\text { thermal } \\
\text { capacity } \\
{[\mathrm{J} / \mathrm{kg} \mathrm{K}]}\end{array}$ & $\begin{array}{l}\text { Specific } \\
\text { volume } \\
{\left[\mathrm{m}^{3} / \mathrm{kg}\right]}\end{array}$ \\
\hline Aluminium & 660 & 910 & 0.000369 \\
\hline Beryllium & 1285 & 1830 & 0.000543 \\
\hline Copper & 1084 & 390 & 0.000112 \\
\hline Steel, stainless & 1510 & 450 & 0.000134 \\
\hline Titanium & 1670 & 540 & 0.000222 \\
\hline
\end{tabular}




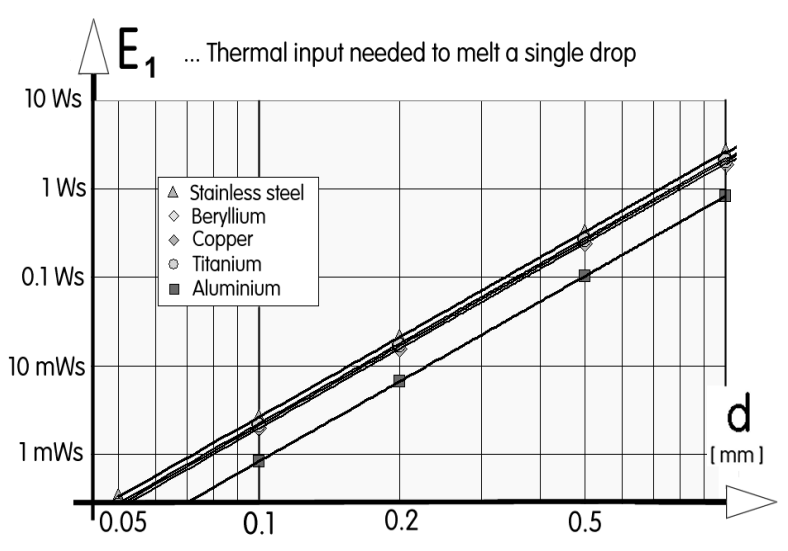

Figure 24. Order-of-magnitude diagram for evaluation of energy to be delivered into solid spheres of various size so as to convert them into the liquid drops. Interestingly, the requirements are nearly the same for many metals.

presented in the accompanying table. A somewhat surprising fact visible in Fig. 24 is the critical dependence of heat transfer on the metal droplet diameter $d$ - while the dependence on the sort of the metal is rather small, almost negligible for the metals of interest (despite the quite wide range of values of the properties in the table on the previous page). Also the order of magnitude spread of the necessary thermal input into the droplet may be interesting, from less than $1 \mathrm{mWs}$ on one side to full one joule $(\mathrm{J}=\mathrm{Ws})$ and more for larger drops.

It is interesting to note in the next Fig. 25 how large power densities are actually needed for melting a modelled stainless steel spherical drop partly irradiated, as described in Fig. 23. To overcome the problem of some of the power transfer radiation impinging at a very small incidence angle (and hence reflected rather than absorbed), the model used for the evaluation according to Figs. 23 and 25 considers only absorption on surface area diameter equal to only one half of the overall droplet diameter $\mathrm{d}$. The irradiated surface is considered ideally black, absorbing all incident radiation. The heating is assumed applied in a pulse lasting for the whole droplet formation time (determined by the formation frequency f). As seen in the diagram Fig. 25, the required thermal power density values for melting the metal may be very high - but fortunately lower than the limits discussed above in Sect. 4 and summarised in Fig. 26.

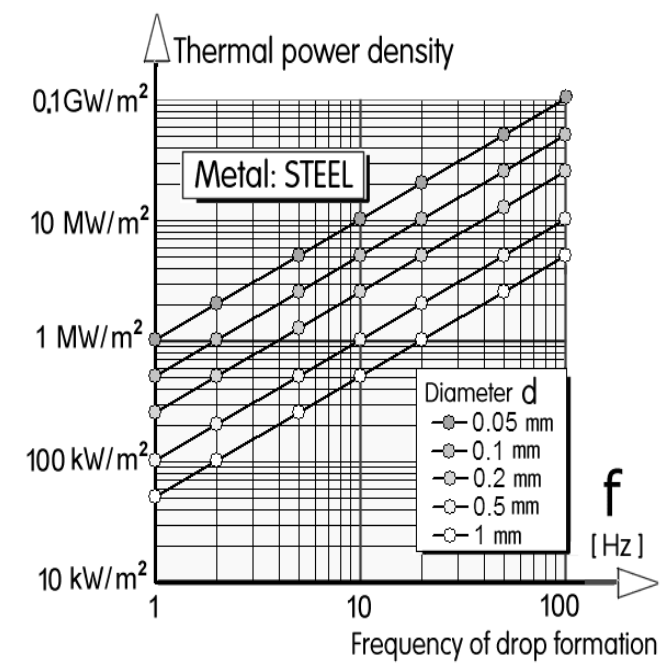

Figure 25. Surface density of irradiation power needed to melt a steel drop of various diameters generated at a particular formation frequency - using the model of Fig. 23.

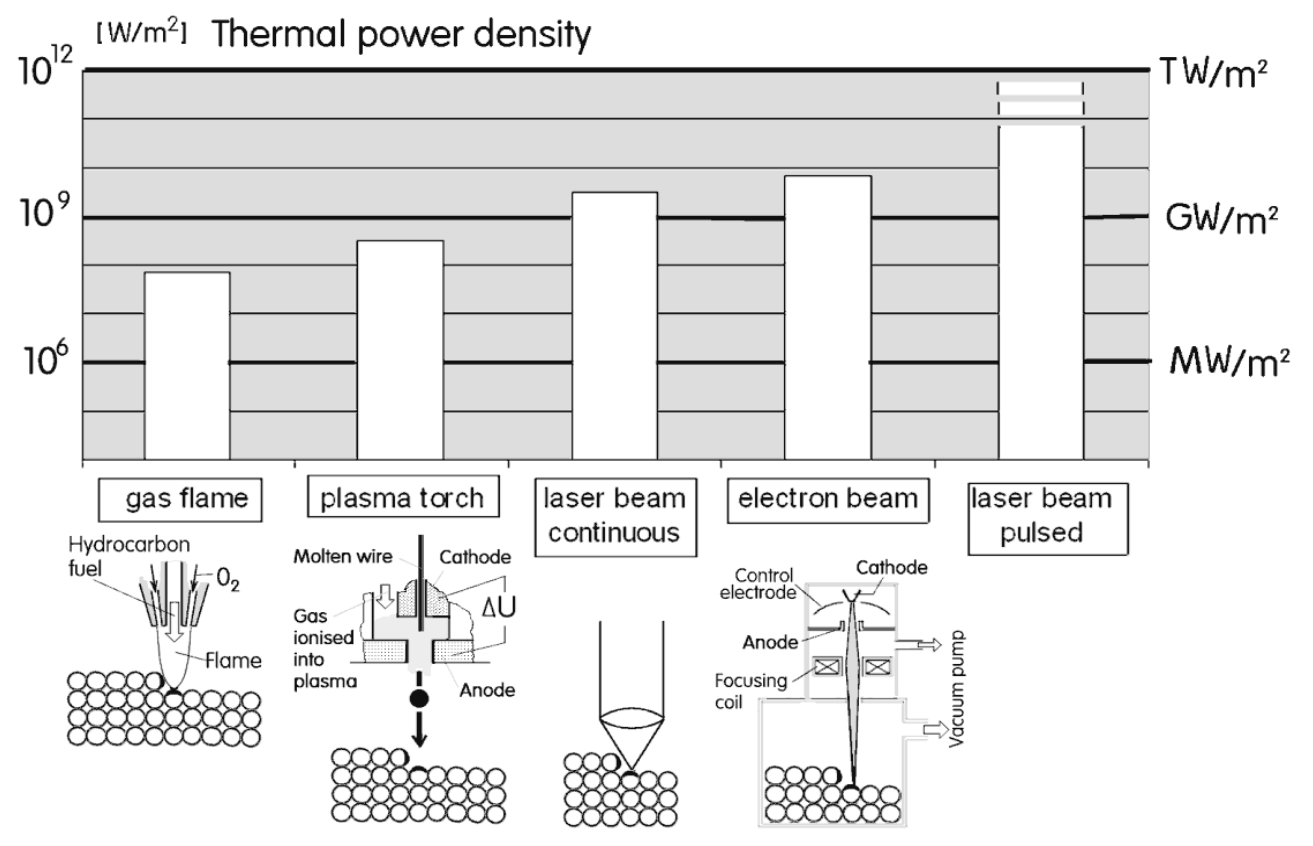

Figure 26. Thermal power densities obtainable with various heat delivering beams. Laser light and electron beams can react sufficiently fast to increase the power by concentrating the output into short pulses. 


\section{Conclusions}

This paper presents, at an introductory level, a general survey of the principles on which is based the recently popular additive manufacturing. The discussion focuses on problem areas that call for deeper investigation. The key problem is low production speed due to the small size of the added droplets and limited repetition rate of their formation. The small droplet size, of course, is necessary for obtaining good quality of produced surfaces - and is also desirable as it decreases the necessary thermal power delivered into the melted droplet. Improvement in productivity needs paying attention to deeper understanding of the processes in the droplets. Some useful information is obtained from earlier studies directed at inkjet printing; nevertheless the molten metal brings its own problems. Some research areas mentioned in this paper are ballistics of controlled flight, undesirable formation of satellite droplets, melted surface oscillation, internal flowfield inside the droplet, and the heat and mass evaporative loss. Most of these problems are of fluid-mechanical and thermokinetic nature.

\section{Acknowledgements}

Author obtained support from GAČR - Grant Agency of the Czech Republic, by research grant Nr. 13-23046S. There was also institutional support RVO:61388998.

\section{References}

1. V. Tesar̆, Sensors and Actuators A: Physical 220 1 (2014)

2. W. Ohnesorge, Journal of Applied Mathematics and Mechanics 16, 355 (1936)

3. B. Derby, Annual Review of Material Research 40395 (2010)

4. B. M. Michaelis et al., International Journal of Heat and Mass Transfer 504554 (2007)

5. A. Amirzadeh, M. Raessi, S. Chandra, Experimental Thermal and Fluid Science 4726 (2013)

6. Gage R., US Patent No. US2806124, filed July 1955

7. J. A. Browning, US Patent No. 4370538, filed May 1980

8. M. N. Butova, US Patent No. 3930139, filed May 1974

9. V. I. Savin et al., Izvestiya Akademii Nauk SSSR, Metally, Nov.-Dec. 1979

10. C.K.N. Patel, Physical Review 136 A1187 (1964)

11. J.-P. Negel J.-P., SPIE Newsroom, (Aug. 2014)

12. I. Mingareev et al., Proc. SPIE8970 Conf. on Laser 3D Manufacturing, (Mar. 2014)

13. M. von Pirani, Journal of Physics D: Applied Physics 45175202 (2012)

14. V. Tesař V., Chemical Engineering Journal, 235 368 (2013)

15. J. M. Hoey et al., Journal of Nanotechnology, 2012 Article ID 324380 (2012)
16. Š. Šikalo , E.N. Ganič, Experimental Thermal and Fluid Science 3197 (2006)

17. K.-L. Pan, C.-Y. Hung, Journal of Colloid and Interface Science 352186 (2010)

18. B. Bachmann, E. Siewert, J. Schein, Journal of Physics D: Applied Physics, 45175202 (2012)

19. Y. S. Oh et al., International Journal of Heat and Fluid Flow $\mathbf{5 0} 27$ (2014)

20. E. J. Vega et al., International Journal of Advanced Manufacturing Technology 701395 (2014)

21. M.P. Mughal, H. Fawad, R. Mufti, Heat and Mass Transfer, 42226 (2006)

22. G. Trapaga et al., Metallurgical Transactions $B$ 23B 7101 (1992)

23. N. Lass et al., Micromachines 449 (2013)

24. V. Tesar̆, Chemical Engineering Science 116 843 (2014) 